\title{
Quasi-isometric embeddings into diffeomorphism groups
}

\author{
Michael Brandenbursky and Jarek Kędra
}

\begin{abstract}
Let $M$ be a smooth compact connected oriented manifold of dimension at least two endowed with a volume form. Assuming certain conditions on the fundamental group $\pi_{1}(M)$ we construct quasi-isometric embeddings of either free Abelian or direct products of non-Abelian free groups into the group of volume preserving diffeomorphisms of $M$ equipped with the $L^{p}$ metric induced by a Riemannian metric on $M$.
\end{abstract}

Mathematics Subject Classification (2010). 57S05, $20 \mathrm{~F} 65$.

Keywords. Groups of diffeomorphisms, $L^{p}$-metrics, quasi-isometric embeddings, distortion.

\section{Introduction}

1.A. The $L^{p}$-metric. Let $M$ be a compact connected and oriented Riemannian manifold and let $\operatorname{Diff}(M, \mu)$ denote the group of $C^{r}$-diffeomorphisms of $M$ acting by the identity on a neighborhood of the boundary and preserving the volume form $\mu$ induced by the metric. Unless otherwise stated we assume that $\operatorname{Diff}(M, \mu)$ is equipped with the $C^{k}$-topology for some fixed $1 \leq k \leq r \leq \infty$.

In the present paper we study the geometry of the identity component $\operatorname{Diff}_{0}(M, \mu)$ of the above group endowed with the right invariant $L^{p}$-metric. It is defined as follows. Let

$$
\mathscr{L}_{p}\left\{g_{t}\right\}:=\int_{0}^{1} d t\left(\int_{M}\left|\dot{g}_{t}(x)\right|^{p} \mu\right)^{\frac{1}{p}}
$$

be the $L^{p}$-length of a smooth isotopy $\left\{g_{t}\right\}_{t \in[0,1]} \subset \operatorname{Diff}_{0}(M, \mu)$, where $\left|\dot{g}_{t}(x)\right|$ denotes the length of the tangent vector $\dot{g}_{t}(x) \in T_{x} M$ induced by the Riemannian metric. Observe that this length is right-invariant, that is, $\mathscr{L}_{p}\left\{g_{t} \circ f\right\}=\mathscr{L}_{p}\left\{g_{t}\right\}$ for any $f \in \operatorname{Diff}(M, \mu)$. It defines a right-invariant metric on $\operatorname{Diff}_{0}(M, \mu)$ by

$$
\mathbf{d}_{p}\left(g_{0}, g_{1}\right):=\inf _{g_{t}} \mathscr{L}_{p}\left\{g_{t}\right\}
$$

where the infimum is taken over all paths from $g_{0}$ to $g_{1}$.

If $p=2$ then the group $\operatorname{Diff}_{0}(M, \mu)$ is in fact equipped with a Riemannian metric inducing the above $L^{2}$-length. The geodesics of this metric are the solutions of the 
equations of the flow of an incompressible fluid [1], which makes the $p=2$ case the most interesting. It is known that if $M$ is a simply connected Riemannian manifold of dimension at least three then the $L^{2}$-diameter of the group $\operatorname{Diff}_{0}(M, \mu)$ is finite [18]. On the other hand Eliashberg and Ratiu [10] proved that this diameter is infinite for surfaces and for manifolds with positive first Betti number. See Arnol'd-Khesin [2] and Khesin-Wendt [14], Section 3.6, for a detailed discussion.

Remark 1.1. The property of preserving the volume is essential to prove the right invariance of the above metric. One can define the $L^{p}$-metric by defining first a norm of a diffeomorphism $g$ by

$$
\|g\|_{p}:=\inf _{g_{t}} \mathscr{L}_{p}\left\{g_{t}\right\}
$$

where the infimum is taken over all isotopies from the identity to $g$. Then the metric $\mathbf{d}_{p}(g, h):=\left\|g h^{-1}\right\|_{p}$ is right invariant by definition. However, in order to prove the triangle inequality it is necessary to use the property of preserving the volume.

1.B. The main result. A map $\psi:\left(X_{1}, \mathrm{~d}_{1}\right) \rightarrow\left(X_{2}, \mathrm{~d}_{2}\right)$ between metric spaces is called large scale Lipschitz [17], Remark 1.9, if there exist constants $A, B \geq 0$ such that

$$
\mathrm{d}_{2}(\psi(x), \psi(y)) \leq A \cdot \mathrm{d}_{1}(x, y)+B .
$$

Let $m \in M$ be a reference point. Let $\operatorname{ev}_{m}: \operatorname{Diff}_{0}(M, \mu) \rightarrow M$ be the evaluation map defined by $\operatorname{ev}_{m}(f):=f(m)$ and let $G_{\mu} \subset \pi_{1}(M)$ be the image of the homomorphism induced by ev $\mathrm{e}_{m}$. It is easy to prove that $G_{\mu}$ is contained in the center of $\pi_{1}(M)$. The subgroup $G_{\mu}$ is called the Gottlieb group associated with the volume form $\mu$ because the groups of similar origin were first studied by Gottlieb in [11].

Let $\operatorname{Diff}(M, \mu, m) \subset \operatorname{Diff}_{0}(M, \mu)$ be the isotropy of the reference point $m$. Let us define a homomorphism

$$
\Phi: \operatorname{Diff}(M, \mu, m) \rightarrow \pi_{1}(M) / G_{\mu}
$$

as follows. Let $g \in \operatorname{Diff}(M, \mu, m)$ and let $\left\{g_{t}\right\} \subset \operatorname{Diff}_{0}(M, \mu)$ be a smooth isotopy from the identity to $g$. The value $\Phi(g)$ is represented by the loop $\left\{g_{t}(m)\right\}$ and it is straightforward to show that $\Phi$ is well defined.

Let $B(m, r) \subset M$ be a ball of radius $r>0$ centered at a reference point $m \in M$. Let $\operatorname{Diff}(M, \mu, B(m, r))$ denote the subgroup of $\operatorname{Diff}_{0}(M, \mu)$ consisting of diffeomorphisms preserving the ball $B(m, r)$ pointwise. The metric on the group $\operatorname{Diff}(M, \mu, B(m, r))$ is induced from the $L^{p}$-metric on $\operatorname{Diff}_{0}(M, \mu)$. The following is our main technical result which is proven in Section 2.

Theorem 1.2. Let $M$ be a compact connected and oriented Riemannian manifold. For all small enough $r>0$ the homomorphism

$$
\Phi: \operatorname{Diff}(M, \mu, B(m, r)) \rightarrow \pi_{1}(M) / G_{\mu}
$$

is surjective and large scale Lipschitz with respect to the $L^{p}$-metric on the group $\operatorname{Diff}(M, \mu, B(m, r))$ and the word metric on $\pi_{1}(M) / G_{\mu}$. 
Recall that the word norm on a group $\Gamma$ generated by a symmetric finite set $S \subset \Gamma$ is defined by

$$
|\gamma|_{S}:=\min \left\{k \in \mathbb{N} \mid \gamma=s_{1} \ldots s_{k} \text { where } s_{i} \in S\right\} .
$$

The word metric is defined by $\mathrm{d}_{S}\left(\gamma_{1}, \gamma_{2}\right):=\left|\gamma_{1}\left(\gamma_{2}\right)^{-1}\right|_{S}$. It is right-invariant and it depends on the choice of a finite generating set up to a quasi-isometry (defined below) [5], Example 8.17.

1.C. Applications. Let $\left(X_{1}, \mathrm{~d}_{1}\right)$ and $\left(X_{2}, \mathrm{~d}_{2}\right)$ be two metric spaces. A function $f: X_{1} \rightarrow X_{2}$ is a quasi-isometric embedding if there exist two constants $A \geq 1$ and $B \geq 0$ such that

$$
\frac{1}{A} \mathrm{~d}_{1}(x, y)-B \leq \mathrm{d}_{2}(f(x), f(y)) \leq A \mathrm{~d}_{1}(x, y)+B .
$$

In the case when $\left(X_{1}, \mathrm{~d}_{1}\right)$ and $\left(X_{2}, \mathrm{~d}_{2}\right)$ are metric groups, we require $f$ to be an injective homomorphism. We say that $f$ is a quasi-isometry if, in addition to (1), there exists a constant $C \geq 0$ such that for every $u \in X_{2}$ there exists $x \in X_{1}$ with the property

$$
\mathrm{d}_{2}(u, f(x)) \leq C .
$$

Let $\Gamma$ be a group equipped with the word metric associated with a finite generating set $S \subset \Gamma$. An element $\gamma$ is called undistorted in $\Gamma$ if there exists a positive constant $C>0$ such that

$$
\left|\gamma^{n}\right|_{S} \geq C \cdot n \text {. }
$$

Otherwise, $\gamma$ is called distorted. These properties do not depend on the choice of a finite generating set.

Theorem 1.3. Let $M$ be a compact connected and oriented Riemannian manifold of dimension at least two. If $\pi_{1}(M) / G_{\mu}$ contains an undistorted element then $\left.\operatorname{(Diff}_{0}(M, \mu), \mathbf{d}_{p}\right)$ contains quasi-isometrically embedded free Abelian group of an arbitrary rank. In particular, the metric group $\left(\operatorname{Diff}_{0}(M, \mu), \mathbf{d}_{p}\right)$ has infinite diameter.

This result generalizes a theorem of Eliashberg and Ratiu [10] where they prove the infiniteness of the diameter under the assumption that the first Betti number of $M$ is positive and the center of the fundamental group is trivial. Notice that there exist compact oriented manifolds with the fundamental group isomorphic to an arbitrary finitely presented group. Such a group can be chosen to have finite abelianization (hence the first Betti number is equal to zero) and usually groups have undistorted elements. For example, $\operatorname{SL}(2, \mathbb{Z})$ has finite abelianization. Moreover, if $g \in \operatorname{SL}(2, \mathbb{Z})$ has an eigenvalue $\lambda$ such that $|\lambda| \neq 1$ then the cyclic group generated by $g$ is undistorted. 
In fact, no finitely presented group with all its elements distorted is known. On the other hand, Osin [16] constructs an example of an infinite finitely generated group with exactly two conjugacy classes. In particular, such a group does not have undistorted elements.

Theorem 1.4. Let $M$ be a compact connected and oriented Riemannian manifold of dimension at least three. If $\pi_{1}(M) / G_{\mu}$ contains quasi-isometrically embedded nonAbelian free group then $\left(\operatorname{Diff}_{0}(M, \mu), \mathbf{d}_{p}\right)$ contains quasi-isometrically embedded direct product of any finite number of free groups of arbitrary ranks.

Essentially, the idea of the proof is to appropriately embed disjoint copies of the figure eight into $M$ and suitably apply Theorem 1.2. Thus the situation is a bit different for surfaces, where we get the following slightly weaker statement.

Theorem 1.5. Let $\Sigma_{g, k}$ be a compact connected and oriented surface of genus $g$ with $k$ boundary components. Then $\left(\operatorname{Diff}_{0}\left(\Sigma_{g, k}, \mu\right), \mathbf{d}_{p}\right)$ contains quasi-isometrically embedded direct product of $2 g+k-2$ copies of finitely generated non-Abelian free groups of arbitrary ranks.

Remark 1.6. Let $\mathbb{D}^{2}$ be a unit Euclidean disc in $\mathbb{R}^{2}$, i.e. in our notation $\mathbb{D}^{2}$ is diffeomorphic to $\Sigma_{0,1}$. In [3] Benaim and Gambaudo showed, using a different method, that the group (Diff $\left.\left(\mathbb{D}^{2}, \mu\right), \mathbf{d}_{p}\right)$ contains quasi-isometrically embedded finitely generated free or free Abelian group of arbitrary rank. Crisp and Wiest proved the same fact for planar right-angled Artin groups [7]. We also would like to mention that M. Kapovich proved that any right-angled Artin group embeds into the group of Hamiltonian diffeomorphisms of any symplectic manifold $(M, \omega)$, see [12]. However, it is not known whether the embedding he constructs in [12] is quasi-isometric with respect to $L^{p}$-metric.

\section{D. Examples}

Example 1.7. Since the Artin pure braid group $\mathbf{P}_{3}$ on three strands is isomorphic to $\mathbb{F}_{2} \times \mathbb{Z}$ (see the proof of Theorem 1.16 in [13]) it embeds quasi-isometrically into $\left.\operatorname{(Diff}_{0}(M, \mu), \mathbf{d}_{p}\right)$, where the manifold $M$ is as in Theorem 1.4.

Example 1.8. If a subgroup $\Gamma$ of a direct product of $n$ free groups has finitely generated homology up to degree $n$ then $\Gamma$ contains a finite index subgroup isomorphic to a direct product of at most $n$ free groups [6]. On the other hand, there are examples of finitely presented subgroups of a direct product of free groups which have more complicated finiteness properties. More concretely, the kernel $\Gamma$ of the homomorphism $\left(\mathbb{F}_{2}\right)^{n} \rightarrow \mathbb{Z}$ sending each generator to one is of type $\mathrm{FP}_{n}$ but not $\mathrm{FP}_{n+1}$. These examples are known as Bieri-Stallings groups, see Bestvina-Brady [4], Example 6.3.

Suppose that $\Gamma \rightarrow \mathbb{F}_{2} \times \cdots \times \mathbb{F}_{2}$ is the inclusion of a finitely presented BieriStallings group. It follows from the proof of Theorem 11.7 of Dison's thesis [9] that 
this inclusion is a quasi-isometric embedding. Indeed, since the quotient is isomorphic to $\mathbb{Z}$, its isoperimetric function is linear and hence, by Dison's Lemma 9.5 in [9] the distortion function of the above inclusion is linear. A more straightforward proof can be found in Bridson-Haefliger [5], Exercise 5.12 (3).

Recall that if $\psi: H \rightarrow G$ is an injective homomorphism of metric groups then its distortion function $\Delta: \mathbb{R}_{+} \rightarrow \mathbb{R}_{+}$is defined by

$$
\Delta(r):=\max \left\{\mathrm{d}_{H}(1, h) \mid \mathrm{d}_{G}(1, \psi(h))<r\right\} .
$$

Observe that the distortion function is linear if and only if $\psi$ is a quasi-isometric embedding.

Remark 1.9. Let $M=\mathbb{T}^{n}$ be the $n$-dimensional torus equipped with a volume form $\mu$. Neither the result of Eliashberg and Ratiu nor our Theorem 1.3 apply to $\operatorname{Diff}\left(\mathbb{T}^{n}, \mu\right)$ because $G_{\mu}=\pi_{1}\left(\mathbb{T}^{n}\right)$ and the whole group is central.

Example 1.10. Let $M \subset \mathbb{R}^{3}$ be a closed domain with free non-Abelian fundamental group. The geometry of $\left(\operatorname{Diff}_{0}(M, \mu), \mathbf{d}_{p}\right)$ models the behavior of an incompressible fluid filling a tank of the shape of $M$. Theorem 1.4 describes a large scale complexity of mixing such a fluid.

1.E. The symplectic case. If $(M, \omega)$ is a symplectic manifold, then the group $\operatorname{Diff}_{0}(M, \mu)$ in all the results above can be replaced either by the group $\operatorname{Symp}_{0}(M, \omega)$ of symplectic diffeomorphisms isotopic to the identity, or by the group $\operatorname{Ham}(M, \omega)$ of Hamiltonian diffeomorphisms, see Remark 2.2 in the proof of Theorem 1.2.

\section{Proofs}

2.A. An abstract lemma. Let $\left(G, \mathrm{~d}_{G}\right)$ be a metric group with the identity element $1_{G}$. For $g \in G$ we set

$$
\|g\|_{G}:=\mathrm{d}_{G}\left(1_{G}, g\right) \quad \text { and } \quad \operatorname{diam}(G):=\sup _{g \in G}\|g\|_{G} .
$$

Lemma 2.1. Let $\left(G, \mathrm{~d}_{G}\right),\left(H, \mathrm{~d}_{H}\right),\left(K, \mathrm{~d}_{K}\right)$ be three metric groups, such that $H$ is finitely generated and $\mathrm{d}_{H}$ is a word metric w.r.t. some finite generating set $S$. Suppose that $\Phi: G \rightarrow K$ is a large scale Lipschitz homomorphism. Let $\Psi: H \rightarrow G$ be a homomorphism, such that $\Phi \Psi: H \rightarrow K$ is a quasi-isometric embedding. Then $\Psi$ is a quasi-isometric embedding.

Proof. The homomorphism $\Psi$ is injective, because $\Phi \Psi$ is injective. Let $h \in H$. The homomorphism $\Phi \Psi$ is a quasi-isometric embedding, hence there exist two constants $A_{1} \geq 0$ and $B_{1} \geq 0$ such that

$$
A_{1}\|h\|_{H}-B_{1} \leq\|\Phi \Psi(h)\|_{K}
$$


The homomorphism $\Phi$ is a large scale Lipschitz, hence there exist two constants $A_{2}>0$ and $B_{2}>0$ such that

$$
\|\Phi \Psi(h)\|_{K} \leq A_{2}\|\Psi(h)\|_{G}+B_{2} .
$$

Combining inequalities (2) and (3) we get

$$
\frac{A_{1}}{A_{2}}\|h\|_{H}-\left(\frac{B_{1}+B_{2}}{A_{2}}\right) \leq\|\Psi(h)\|_{G} .
$$

Let $k$ be such that $\|h\|_{H}=k$. The group $H$ is finitely generated, hence $h=$ $h_{1} \ldots h_{k}$, where $h_{i} \in S$ for each $1 \leq i \leq k$. Denote by

$$
M_{\Psi}:=\max \left\{\left\|\Psi\left(h_{1}\right)\right\|_{G}, \ldots,\left\|\Psi\left(h_{k}\right)\right\|_{G}\right\} .
$$

It follows that

$$
\|\Psi(h)\|_{G}=\left\|\Psi\left(h_{1}\right) \ldots \Psi\left(h_{1}\right)\right\|_{G} \leq \sum_{i=1}^{k}\left\|\Psi\left(h_{i}\right)\right\|_{G} \leq M_{\Psi} \cdot k=M_{\Psi}\|h\|_{H} .
$$

Inequalities (4) and (5) conclude the proof of the lemma.

In the proofs below we make use of the fact that the metric on the groups $\operatorname{Diff}(M, \mu, B(m, r))$ and $\operatorname{Diff}\left(M, \mu, \sqcup_{i} B\left(m_{i}, r\right)\right)$ (this group is defined in the proof of Theorem 1.3) is induced from the $L^{p}$-metric on the whole group $\operatorname{Diff}(M, \mu)$.

2.B. Proof of Theorem 1.2. In the first part we prove the surjectivity of the homomorphism $\Phi: \operatorname{Diff}(M, \mu, B(m, r)) \rightarrow \pi_{1}(M) / G_{\mu}$.

Let $S^{\prime}:=\left\{\left[\gamma_{1}\right], \ldots,\left[\gamma_{k}\right]\right\} \subset \pi_{1}(M)$ be a symmetric generating set of the fundamental group of $M$ such that each representative $\gamma_{i}$ is a simple closed curve. It follows from the tubular neighborhood theorem that for each $\gamma_{i}$ there exists $r_{i}>0$, the standard (n-1)-dimensional ball $B_{r_{i}}^{n-1} \subset \mathbb{R}^{n}$ of radius $r_{i}>0$, and a volume-preserving embedding

$$
\mathrm{emb}_{i}: B_{r_{i}}^{n-1} \times \mathbb{S}^{1} \hookrightarrow M
$$

such that emb $\left.\right|_{\{0\} \times \mathbb{S}^{1}}$ is the curve $\gamma_{i}$ and $r_{i}=\mathrm{d}_{M}\left(\gamma_{i},\left.\mathrm{emb}_{i}\right|_{\partial B_{r_{i}}^{n-1} \times \mathbb{S}^{1}}\right)$. The volume form on the product $B_{r_{1}}^{n-1} \times \mathbb{S}^{1}$ is the standard Euclidean volume and $\mathrm{d}_{M}$ denotes the distance on $M$ induced by the Riemannian metric.

Let $r=\min _{1 \leq i \leq k} r_{i}$. Then $\mathrm{emb}_{i}: B_{r}^{n-1} \times \mathbb{S}^{1} \hookrightarrow M$ is volume-preserving for each $i$ and $\left.\mathrm{emb}_{i}\right|_{0 \times \mathbb{S}^{1}}=\gamma_{i}$. It is straightforward to construct a smooth isotopy of volume-preserving diffeomorphisms

$$
g_{t}: B_{r}^{n-1} \times \mathbb{S}^{1} \rightarrow B_{r}^{n-1} \times \mathbb{S}^{1}
$$

between $g_{0}=\mathrm{Id}$ and $g_{1}$ such that: 
- For each $t \in[0,1]$ the diffeomorphism $g_{t}$ equals to the identity in the neighborhood of $\partial B_{r}^{n-1} \times \mathbb{S}^{1}$, and the time-one map $g_{1}$ is equal to the identity on $B_{r^{\prime}}^{n-1} \times \mathbb{S}^{1}$, where $0<r^{\prime}<r$.

- Each diffeomorphism $g_{t}$ preserves the foliation of $B_{r}^{n-1} \times \mathbb{S}^{1}$ by the circles $\{x\} \times \mathbb{S}^{1}$ and for every $x \in B_{r^{\prime}}^{n-1}$ the restriction $g_{t}:\{x\} \times \mathbb{S}^{1} \rightarrow\{x\} \times \mathbb{S}^{1}$ is the rotation by $2 \pi t$. It follows that the time-one map $g_{1}$ equals the identity on $B_{r^{\prime}}^{n-1} \times \mathbb{S}^{1}$.

We identify $B_{r}^{n-1} \times \mathbb{S}^{1}$ with its image with respect to the embedding emb ${ }_{i}$. Then we extend each isotopy $g_{t}$ by the identity on $M \backslash B_{r}^{n-1} \times \mathbb{S}^{1}$ obtaining smooth isotopies $g_{t, i} \in \operatorname{Diff}_{0}(M, \mu)$. This shows that every representative $\gamma_{i}$ of a generator of the fundamental group of $M$ arises as simple closed curve $\left\{g_{t, i}(m)\right\}$ and hence the homomorphism

$$
\Phi: \operatorname{Diff}(M, \mu, B(m, r)) \rightarrow \pi_{1}(M) / G_{\mu}
$$

is surjective.

Remark 2.2. Notice that if $M$ is a symplectic manifold then the above isotopies can be constructed to be Hamiltonian.

Let $\Pi: \pi_{1}(M) \rightarrow \pi_{1}(M) / G_{\mu}$ be the projection homomorphism. Consequently $S:=\Pi\left(S^{\prime}\right)$ is a finite generating set for the quotient $\pi_{1}(M) / G_{\mu}$. Let $\Pi_{M}: M_{\bullet} \rightarrow$ $M$ be the Riemannian covering associated with $\Pi$. This means that the metric on $M \bullet$ is induced from the Riemannian metric on $M$. The corresponding distance will be denoted by d.

Now we shall prove that $\Phi$ is a large scale Lipschitz map. That is, we show that there exist positive constants $A$ and $B$ independent of $g$ such that

$$
A \cdot\|g\|_{p}+B \geq\|\Phi(g)\|_{S},
$$

where $\|g\|_{p}:=\mathbf{d}_{p}(g$, Id $)$ is the $L^{p}$-norm of the diffeomorphism $g$.

Let $g \in \operatorname{Diff}(M, \mu, B(m, r))$ and let $\left\{g_{t}\right\}_{t \in[0,1]} \in \operatorname{Diff}_{0}(M, \mu)$ be an isotopy from the identity to $g$. It follows from the Hölder inequality that $\|g\|_{p} \geq C_{p} \cdot\|g\|_{1}$, where $C_{p}$ is some positive constant independent of $g$. Hence it is enough to prove the statement for $p=1$.

Let $m_{\bullet} \in \Pi_{M}^{-1}(m)$, and let $\left\{g_{\bullet}\left(m_{\bullet}\right)\right\}$ be the lift of $\left\{g_{t}(m)\right\}$ starting at the point $m_{\bullet}$. The manifold $M$ is compact, hence by the Švarc-Milnor lemma [5], [15], the inclusion of the orbit of $m_{\bullet}$ with respect to the deck transformation group $\pi_{1}(M) / G_{\mu}$ defines a quasi-isometry

$$
\pi_{1}(M) / G_{\mu} \stackrel{\text { q.i. }}{\simeq}\left(M_{\bullet}, \mathrm{d}_{\bullet}\right) .
$$

In particular, it means that there exist positive constants $A^{\prime}, B^{\prime}$ such that

$$
d_{\bullet}\left(m_{\bullet}, g_{\bullet}, 1\left(m_{\bullet}\right)\right) \geq A^{\prime}\|\Phi(g)\|_{S}-B^{\prime} .
$$


Let $x \in B(m, r) \subset M$. We claim that the length of the flow-line $g_{t}(x)$ is bounded by the distance $\mathrm{d}_{\bullet}\left(m_{\bullet}, g_{\bullet, 1}\left(m_{\bullet}\right)\right)$ up to the diameter of the ball. To see this, consider the lift of each flow-line $g_{t}(x)$ starting at a ball of radius $r$ in $M_{\bullet}$ centered at $m_{\bullet}$ and observe that such a lift ends in a ball of radius $r$ centered at $g_{\bullet, 1}\left(m_{\bullet}\right)$. Indeed, let $\alpha:[0,1] \rightarrow B(m, r)$ be a path between $x$ and $m$. Then the map

$$
H:[0,1] \times[0,1] \rightarrow M,
$$

defined by $H(t, s)=g_{t}(\alpha(s))$, is a homotopy from $\left\{g_{t}(m)\right\}$ to $\left\{g_{t}(x)\right\}$. Lifting this homotopy shows that the lift of $\left\{g_{t}(x)\right\}$ ends at the ball $B\left(g_{\bullet, 1}\left(m_{\bullet}\right), r\right)$. Finally, we obtain that

$$
\operatorname{Length}\left(g_{t}(x)\right):=\int_{0}^{1}\left|\dot{g}_{t}(x)\right| d t \geq \mathrm{d}_{\bullet}\left(m_{\bullet}, g_{\bullet, 1}\left(m_{\bullet}\right)\right)-2 r .
$$

as claimed. Combining inequalities (6) and (7) we get that

$$
\operatorname{Length}\left(g_{t}(x)\right) \geq A^{\prime}\|\Phi(g)\|_{S}-\left(B^{\prime}+2 r\right)
$$

for every $x \in B(m, r)$. Hence by Fubini theorem and the above inequality we have

$$
\begin{aligned}
\mathscr{L}_{1}\left(\left\{g_{t}\right\}\right) & =\int_{0}^{1} d t\left(\int_{M}\left|\dot{g}_{t}(x)\right| \mu\right) \\
& =\int_{M} \mu\left(\int_{0}^{1}\left|\dot{g}_{t}(x)\right| d t\right) \\
& \geq \operatorname{vol}(B(m, r)) \cdot \min _{x \in B(m, r)} \operatorname{Length}\left(g_{t}(x)\right) \\
& \geq \operatorname{vol}(B(m, r)) \cdot A^{\prime}\|\Phi(g)\|_{S}-\operatorname{vol}(B(m, r)) \cdot\left(B^{\prime}+2 r\right) .
\end{aligned}
$$

Since the above inequalities hold for any isotopy $\left\{g_{t}\right\}_{t \in[0,1]}$ between the identity and $g$, we obtain that

$$
\|\Phi(g)\|_{S} \leq A \cdot\|g\|_{p}+B
$$

where $A=\left(C_{p} \cdot \operatorname{vol}(B(m, r))^{-1} \cdot A^{\prime}\right.$ and $B=\frac{B^{\prime}+2 r}{A^{\prime}}$ and this concludes the proof.

2.C. Proof of Theorem 1.3. Let $n \in \mathbb{N}$ be a positive integer. Recall that we need to prove that there exists a quasi-isometric embedding of a free Abelian group of rank $n$ into $\operatorname{Diff}_{0}(M, \mu)$.

Assume first that the dimension of $M$ is at least three. Let $m_{1}, \ldots, m_{n}$ be distinct points in the interior of $M$ and let $r>0$ be such that the balls $B\left(m_{i}, r\right)$ of radius $r$ centered at $m_{i}$ are pairwise disjoint. Let $\gamma_{i, j}$ be simple closed curves representing the generators of $\pi_{1}\left(M, m_{i}\right)$. We also assume that $\gamma_{i_{1}, j_{1}}$ is disjoint from $\gamma_{i_{2}, j_{2}}$ whenever $i_{1} \neq i_{2}$. We choose $r$ small enough such that the tubular neighborhood of radius $r$ of the above generators are disjoint. 
We denote by $G_{i} \subset \pi_{1}\left(M, m_{i}\right)$ be the corresponding Gottlieb group. The groups $\pi_{1}\left(M, m_{i}\right) / G_{i}$ are pairwise isomorphic. Let $\gamma_{i} \in \pi_{1}\left(M, m_{i}\right) / G_{i}$ be an undistorted element which exists according to the hypothesis. Let

$$
h: \mathbb{Z}^{n} \rightarrow \pi_{1}\left(M, m_{1}\right) / G_{1} \times \cdots \times \pi_{1}\left(M, m_{n}\right) / G_{n}
$$

be the homomorphism defined by

$$
h\left(k_{1}, \ldots, k_{n}\right):=\left(\gamma_{1}^{k_{1}}, \ldots, \gamma_{n}^{k_{n}}\right) .
$$

It immediately follows from the fact that each $\gamma_{i}$ is undistorted that $h$ is a quasiisometric embedding. Let $\operatorname{Diff}\left(M, \mu, \sqcup_{i} B\left(m_{i}, r\right)\right) \subset \operatorname{Diff}_{0}(M, \mu)$ be the subgroup consisting of diffeomorphisms preserving the disjoint union of balls $B\left(m_{i}, r\right)$ pointwise. Let

$$
\Phi_{i}: \operatorname{Diff}\left(M, \mu, B\left(m_{i}, r\right)\right) \rightarrow \pi_{1}\left(M, m_{i}\right) / G_{i}
$$

be the homomorphism defined in Theorem 1.2. Consider a homomorphism

$$
\widetilde{\Phi}: \operatorname{Diff}\left(M, \mu, \sqcup_{i} B\left(m_{i}, r\right)\right) \rightarrow \pi_{1}\left(M, m_{1}\right) / G_{1} \times \cdots \times \pi_{1}\left(M, m_{n}\right) / G_{n}
$$

which is the composition of the (diagonal) inclusion

$$
\iota: \operatorname{Diff}\left(M, \mu, \sqcup_{i} B\left(m_{i}, r\right)\right) \hookrightarrow \prod_{i} \operatorname{Diff}\left(M, \mu, B\left(m_{i}, r\right)\right)
$$

followed by the product homomorphism

$$
\prod_{i} \Phi_{i}: \prod_{i} \operatorname{Diff}\left(M, \mu, B\left(m_{i}, r\right)\right) \rightarrow \prod_{i} \pi_{1}\left(M, m_{i}\right) / G_{i} .
$$

Since the inclusion $\iota$ is an isometric embedding and the $\prod_{i} \Phi_{i}$ is large scale Lipschitz, according to Theorem 1.2, we obtain that $\widetilde{\Phi}$ is a large scale Lipschitz homomorphism.

Let $g_{i} \in \operatorname{Diff}\left(M, \mu, B\left(m_{i}, r\right)\right)$ be an element such that $\Phi_{i}\left(g_{i}\right)=\gamma_{i}$ and $g_{i}$ is supported in the union of the tubular neighborhoods of the loops representing the generators of $\pi_{1}\left(M, m_{i}\right)$ constructed in the beginning of the proof. It follows that the supports of $g_{i}$ and $g_{j}$ are disjoint if $i \neq j$. The existence of $g_{i}$ follows from the proof of Theorem 1.2. Let

$$
\Psi: \mathbb{Z}^{n} \rightarrow \operatorname{Diff}\left(M, \mu, \sqcup_{i} B\left(m_{i}, r\right)\right) \subset \operatorname{Diff}_{0}(M, \mu)
$$

be defined by

$$
\Psi\left(k_{1}, \ldots, k_{n}\right):=g_{1}^{k_{1}} \circ \cdots \circ g_{n}^{k_{n}} .
$$

It is well defined because $g_{i}$ have pair-wise disjoint supports. Recall that we have that

$$
h=\widetilde{\Phi} \circ \Psi: \mathbb{Z}^{n} \rightarrow \prod_{i} \pi_{1}\left(M, m_{i}\right) / G_{i}
$$


and we know that $h$ is a quasi-isometric embedding and $\widetilde{\Phi}$ is large scale Lipschitz. Consequently the map $\Psi$ is a quasi-isometric embedding according to Lemma 2.1.

Let us now consider the two-dimensional case. Let $M=\Sigma_{g, k}$ be a compact oriented surface of genus $g$ with $k$ boundary components. Observe that $\pi_{1}\left(\Sigma_{g, k}\right) / G_{\mu}$ is trivial if either $g=0$ and $k \leq 2$ or $g=1$ and $k=0$. Otherwise it is either free non-Abelian group or the fundamental group of a closed oriented surface. In each case it is straightforward to define an embedding

$$
\text { emb: } \mathbb{S}^{1} \times[0,2 n] \rightarrow M
$$

in such a way that each loop emb $\left(\mathbb{S}^{1} \times\{t\}\right)$ represents an undistorted element in $\pi_{1}(M, \mathrm{emb}(1, t))$. Let $g_{i}: M \rightarrow M$, for $i=1, \ldots, n$ be an area preserving diffeomorphism satisfying each of the following conditions:

- it is supported in emb $\left(\mathbb{S}^{1} \times(2 i-2,2 i)\right)$;

- it preserves the ball emb $\left(B_{i}\right)$, where $B_{i} \subset \mathbb{S}^{1} \times[0,2 n]$ is a ball of diameter one centered at $(1,2 i-1)$;

- it is the time one map of an isotopy from the identity which acts as the full rotation on the loop emb $\left(\mathbb{S}^{1} \times\{2 i-1\}\right)$.

As in the previous part the homomorphism $\Psi: \mathbb{Z}^{n} \rightarrow \operatorname{Diff}_{0}(M, \mu)$ defined by $\Psi\left(k_{1}, \ldots, k_{n}\right):=g_{1}^{k_{1}} \circ \cdots \circ g_{n}^{k_{n}}$ is the required quasi-isometric embedding.

2.D. Proof of Theorem 1.4. This proof is a modification of the proof of Theorem 1.3 for three dimensional $M$ where the cyclic group $\mathbb{Z}$ is replaced by a non-Abelian free group $\mathbb{F}_{2}$ on two generators. More precisely, let $f_{i}, g_{i} \in \operatorname{Diff}_{0}(M, \mu)$ be diffeomorphisms satisfying each of the following conditions (we use here the notation of the proof of Theorem 1.3):

- the support of $f_{i}$ and $g_{i}$ is contained in the neighborhood of the union of the loops $\gamma_{i, j}$

- the free non-Abelian group in $\pi_{1}\left(M, m_{i}\right) / G_{i}$ is generated by the images $\Phi_{i}\left(f_{i}\right)$ and $\Phi_{i}\left(g_{i}\right)$.

Such diffeomorphisms can be constructed in a similar way as $g_{i}$ 's in the proof of Theorem 1.3.

Let $w \in \mathbb{F}_{2}$ be a reduced word and given two elements $f, g \in \operatorname{Diff}(M, \mu)$ let $w(f, g)$ denote the induced diffeomorphism of $M$. Let

$$
\Psi: \mathbb{F}_{2} \times \cdots \times \mathbb{F}_{2} \rightarrow \operatorname{Diff}\left(M, \mu, \sqcup_{i} B\left(m_{i}, r\right)\right) \subset \operatorname{Diff}_{0}(M, \mu)
$$

be defined by $\Psi\left(w_{1}, \ldots, w_{n}\right):=w_{1}\left(f_{1}, g_{1}\right) \circ \ldots \circ w_{n}\left(f_{n}, g_{n}\right)$. As before $\Psi$ is a quasi-isometric embedding of a product of free groups on two generators into $\operatorname{Diff}_{0}(M, \mu)$. Since $\mathbb{F}_{2}$ contains quasi-isometrically embedded a non-Abelian free group of an arbitrary finite rank [8] the proof is finished. 
2.E. Proof of Theorem 1.5. The proof of the two dimensional case of Theorem 1.3 amounts to constructing a number of disjoint simple closed curves representing an undistorted element in the fundamental group of $M$. The present proof is analogous in the sense that we need to construct an embedding of the disjoint union of $2 g+k-2$ copies of the figure eight into $M$ such that each embedding induces a quasi-isometric embedding $\mathbb{F}_{2} \rightarrow \pi_{1}\left(M, m_{i}\right)$ for $i=1, \ldots, 2 g+k-2$. We leave this straightforward construction as an exercise to the reader.

The rest of the proof is similar to the other proofs. That is, we construct relevant diffeomorphisms $f_{i}, g_{i}$ and observe that the map

$$
\Psi: \mathbb{F}_{2} \times \cdots \times \mathbb{F}_{2} \rightarrow \operatorname{Diff}\left(\Sigma_{g, k}, \mu, \sqcup_{i} B\left(m_{i}, r\right)\right) \subset \operatorname{Diff}_{0}\left(\Sigma_{g, k}, \mu\right)
$$

defined by $\Psi\left(w_{1}, \ldots, w_{n}\right):=w_{1}\left(f_{1}, g_{1}\right) \circ \ldots \circ w_{n}\left(f_{n}, g_{n}\right)$ is a quasi-isometric embedding.

Acknowledgments. We would like to thank Jim Howie and Mark Sapir for answering our questions. Example 1.8 is due to Jim Howie. We also thank Martin Bridson for useful historical comments concerning this example.

This work has been done during the first author's stay in Aberdeen. His visit was supported by the Coleman-Cohen foundation.

\section{References}

[1] V. Arnold, Sur la géométrie différentielle des groupes de Lie de dimension infinie et ses applications à l'hydrodynamique des fluides parfaits. Ann. Inst. Fourier (Grenoble) 16 (1966), 319-361. Zbl 0148.45301 MR 0202082

[2] V. I. Arnold and B. A. Khesin, Topological methods in hydrodynamics. Appl. Math. Sci. 125, Springer-Verlag, New York 1998. Zbl 0902.76001 MR 1612569

[3] M. Benaim and J.-M. Gambaudo, Metric properties of the group of area preserving diffeomorphisms. Trans. Amer. Math. Soc. 353 (2001), 4661-4672. Zbl 0977.57039 MR 1851187

[4] M. Bestvina and N. Brady, Morse theory and finiteness properties of groups. Invent. Math. 129 (1997), 445-470. Zbl 0888.20021 MR 1465330

[5] M. R. Bridson and A. Haefliger, Metric spaces of non-positive curvature. Grundlehren Math. Wiss. 319, Springer-Verlag, Berlin 1999. Zbl 0988.53001 MR 1744486

[6] M. R. Bridson, J. Howie, C. F. Miller, III, and H. Short, Subgroups of direct products of limit groups. Ann. of Math. (2) 170 (2009), 1447-1467. Zbl 1196.20047 MR 2600879

[7] J. Crisp and B. Wiest, Quasi-isometrically embedded subgroups of braid and diffeomorphism groups. Trans. Amer. Math. Soc. 359 (2007), 5485-5503. Zbl 1186.20027 MR 2327038

[8] P. de la Harpe, Topics in geometric group theory. Chicago Lectures in Math., The University of Chicago Press, Chicago 2000. Zbl 0965.20025 MR 1786869 
[9] W. J. Dison, Isoperimetric functions for subdirect products and Bestvina-Brady groups. D.Phil. Thesis, Imperial College, London 2008. www.maths.bris.ac.uk/ mawjd/thesis.pdf

[10] Y. Eliashberg and T. Ratiu, The diameter of the symplectomorphism group is infinite. Invent. Math. 103 (1991), 327-340. Zbl 0725.58006 MR 1085110

[11] D. H. Gottlieb, A certain subgroup of the fundamental group. Amer. J. Math. 87 (1965), 840-856. Zbl 0148.17106 MR 0189027

[12] M. Kapovich, RAAGs in Ham. Geom. Funct. Anal. 22 (2012), 733-755. Zbl 1262.53070 MR 2972607

[13] C. Kassel and V. Turaev, Braid groups. Graduate Texts in Math. 247, Springer, New York 2008. Zbl 1208.20041 MR 2435235

[14] B. Khesin and R. Wendt, The geometry of infinite-dimensional groups. Ergeb. Math. Grenzgeb. (3) 51, Springer-Verlag, Berlin 2009. Zbl 1153.22001 MR 2456522

[15] J. Milnor, A note on curvature and fundamental group. J. Differential Geometry 2 (1968), 1-7. Zbl 0162.25401 MR 0232311

[16] D. Osin, Small cancellations over relatively hyperbolic groups and embedding theorems. Ann. of Math. (2) 172 (2010), 1-39. Zbl 1203.20031 MR 2680416

[17] J. Roe, Lectures on coarse geometry. Univ. Lecture Ser. 31, American Mathematical Society, Providence, RI, 2003. Zbl 1042.53027 MR 2007488

[18] A. I. Shnirelman, Generalized fluid flows, their approximation and applications. Geom. Funct. Anal. 4 (1994), 586-620. Zbl 0851.76003 MR 1296569

Received September 1, 2011; revised May 3, 2012

M. Brandenbursky, Department of Mathematics, 1326 Stevenson Center, Vanderbilt University, Nashville, TN 37240, U.S.A.

E-mail: michael.brandenbursky@ vanderbilt.edu

J. Kędra, Institute of Mathematics, University of Aberdeen, Aberdeen, AB24 3UE, UK

E-mail: kedra@abdn.ac.uk 